\title{
IgA anti-tissue transglutaminase as a diagnostic marker of gluten sensitive enteropathy
}

\author{
R J Lock, M C L Pitcher, D J Unsworth
}

\begin{abstract}
Aims-To compare and contrast the sensitivity, specificity, and positive predictive values of IgA antibodies to guinea pig tissue transglutaminase (ELISA), endomysium, and reticulin (immunofluorescence), and gliadin (ELISA), and IgG antibodies to gliadin and tissue transglutaminase.

Methods-Sera from 27 newly diagnosed patients with coeliac disease, 65 biopsied gastrointestinal disease controls, and 50 consecutive blood donors were tested. All cases were adults.

Results-IgA anti-tissue transglutaminase showed a sensitivity of $85 \%$ (23/27 coeliac disease cases seropositive), specificity $97 \%$ ( $2 / 65$ controls and one blood donor showing low titre positivity), and a positive predictive value of $92 \%$. High titre anti-tissue transglutaminase was only seen in coeliac disease. Disease controls with mucosal damage unrelated to gluten enteropathy were IgA anti-tissue transglutaminase negative. Sensitivity, specificity, and positive predictive values for IgA anti-endomysial antibody (monkey oesophagus) were $100 \%, 100 \%$, and $100 \%$, respectively, and for IgA anti-gliadin, $93 \%, 95 \%$, and $89 \%$, respectively.

Conclusions-Tissue transglutaminase is a major autoantigen in coeliac disease. IgA (but not IgG) anti-tissue transglutaminase, especially when in high titre, is closely associated with coeliac disease, but low titres may not be disease specific. In this small pilot study, the established IgA anti-endomysial assay was the superior test.

(F Clin Pathol 1999;52:274-277)
\end{abstract}

Department of

Immunology,

Southmead Hospital,

Bristol BS10 5NB, UK

R J Lock

D J Unsworth

Department of

Medicine and

Gastroenterology,

Addenbrooke's

Hospital, Cambridge,

UK

M C L Pitcher

Correspondence to:

Dr Unsworth.

email:

joe.unsworth@msmail.nbs. nhs.uk

Accepted for publication 15 December 1998
Keywords: coeliac disease; endomysial antibody; tissue transglutaminase

R1 anti-reticulin (R1-ARA) and antiendomysium (AEA) antibodies of IgA isotype are probably pathognomonic for gluten sensitive enteropathy. ${ }^{12}$ These powerful diagnostic tools have helped show that coeliac disease is underdiagnosed, ${ }^{3}$ can exist as an occult problem in apparently healthy persons, ${ }^{4}$ and can predict gluten sensitive enteropathy in predisposed persons with "latent coeliac disease." The high reliability of these autoantibody tests has led to arguments in favour of screening high risk groups such as relatives and people with type 1 diabetes. Hitherto, the nature of the autoantigens has been unclear and the available immunofluorescence detection methods on tissue sections cumbersome.
The recent report that "tissue transglutaminase" (TT) is a major autoantigen in coeliac disease $^{5}$ is an exciting development. Dieterich et al used patient's AEA to isolate an 85000 molecular weight autoantigen from lysed HT1080 (human sarcoma line) cells by immunoprecipitation. ${ }^{5}$ TT is a calcium dependent enzyme released during tissue injury and capable of cross linking fibronectin and certain collagens. Interestingly, gliadin, which is glutamine-rich, can act as a substrate for TT, and this may explain the observation that gliadin binds selectively to "reticulin" in tissue sections, ${ }^{6}$ possibly autosensitising TT. Guinea pig TT shows close similarity to human TT, is commercially available, and can be used to establish solid phase blood tests (enzyme linked immunosorbent assay, ELISA) to aid the diagnosis of coeliac disease. ${ }^{5}$

To our knowledge, this report is the first attempt to confirm the report of Dieterich et $a l,{ }^{5}$ who only tested a total of 12 coeliac disease cases and seven disease controls. We have used a modified anti-TT ELISA method, omitting the use of bovine serum albumin (BSA) as non-specific blocker, because patients with coeliac disease can develop anti-BSA antibodies. We have compared and contrasted our anti-TT ELISA with established serological screening tests for coeliac disease, including IgA immunofluorescence (AEA, both on monkey oesophagus and human umbilical cord, and R1-ARA), and IgA and IgG anti-gliadin ELISA.

\section{Methods}

PATIENTS AND MATERIALS

All sera were routine samples in the investigation of possible coeliac disease. All patients, including controls with gastrointestinal disease, underwent concurrent small intestinal biopsy. Patients were entered into the study on the basis that they had had a small intestinal biopsy and that there was adequate stored serum, obtained before starting a gluten-free diet. They were otherwise unselected. The majority of the sera were from Addenbrooke's Hospital, Cambridge, collected over an 18 month period. Others were from Bristol and other hospitals that use Southmead Hospital's immunology diagnostic laboratory. Overall, the sera were collected over a five year period and stored in aliquots at $-20^{\circ} \mathrm{C}$. Fifty randomly selected adult blood donor sera (no gut biopsy) were collected and tested anonymously.

Southmead Hospital ethics committee gave approval for use of all the samples.

The 27 coeliac disease cases showed typical histological features of coeliac disease, and sera were tested at presentation, before they started 
Table 1 Summary data showing number of sera positive in each group (percentage in parentheses)

\begin{tabular}{llll}
\hline & Coeliac disease & Disease controls & \multirow{2}{*}{ Blood donors } \\
\hline IgA anti-gliadin & $25 / 27(93 \%)$ & $3 / 65(5 \%)$ & \\
IgG anti-gliadin & $22 / 27(77 \%)$ & $6 / 65(9 \%)$ & \\
Any anti-gliadin & $26 / 27(96 \%)$ & $7 / 65(11 \%)$ & \\
Anti-AEA (monkey oesophagus) & $27 / 27(100 \%)$ & $0 / 65$ & \\
Anti-AEA (umbilical cord) & $26 / 27(96 \%)$ & $1 / 65(2 \%)$ & $0 / 50$ \\
Anti-R1 reticulin & $16 / 27(59 \%)$ & $0 / 65$ & \\
IgA anti-TT & $23 / 27(85 \%)$ & $2 / 65(3 \%)$ & $1 / 50(2 \%)$ \\
IgG anti-TT & $12 / 27(44 \%)$ & $7 / 58(12 \%)$ & $7 / 50(14 \%)$ \\
Any anti-TT & $25 / 27(93 \%)$ & $9 / 58(16 \%)$ & $8 / 50(16 \%)$ \\
\hline
\end{tabular}

AEA, anti-endomysial antibody; TT, tissue transglutaminase.

Table 2 Sensitivity, specificity, and positive predictive value of assays for a diagnosis of untreated coeliac disease when compared with gastrointestinal disease controls

\begin{tabular}{llll}
\hline & Sensitivity & Specificity & Positive predictive value \\
\hline IgA anti-gliadin & $93 \%$ & $95 \%$ & $89 \%$ \\
IgG anti-gliadin & $77 \%$ & $91 \%$ & $79 \%$ \\
Any anti-gliadin & $96 \%$ & $89 \%$ & $79 \%$ \\
Anti-AEA (monkey oesophagus) & $100 \%$ & $100 \%$ & $100 \%$ \\
Anti-AEA (umbilical cord) & $96 \%$ & $98 \%$ & $96 \%$ \\
Anti-R1 reticulin & $59 \%$ & $100 \%$ & $100 \%$ \\
IgA anti-TT & $85 \%$ & $97 \%$ & $92 \%$ \\
IgG anti-TT & $44 \%$ & $88 \%$ & $63 \%$ \\
Any anti-TT & $93 \%$ & $84 \%$ & $74 \%$ \\
\hline
\end{tabular}

AEA, anti-endomysial antibody; TT, tissue transglutaminase.

a gluten-free diet. All 27 patients showed clear clinical and morphological improvement three or more months later on follow up, which included a biopsy.

The 65 adult disease controls were cases who had been investigated for possible coeliac disease. The majority were cases of unexplained anaemia, or suspected malabsorption. Included among these were nine patients with insulin dependent diabetes (type 1) and diarrhoea, 26 with unexplained anaemia, 11 with unexplained diarrhoea, four with chronic tiredness and a family history of coeliac disease, and one case with giardiasis and hypogammaglobulinaemia. The remaining cases had non-specific gastrointestinal complaints including irritable bowel syndrome, abdominal pains, or other symptoms. Eight of the disease controls showed small bowel abnormalities ranging from subtotal villus atrophy in the giardiasis case to non-specific duodenitis in four other cases.

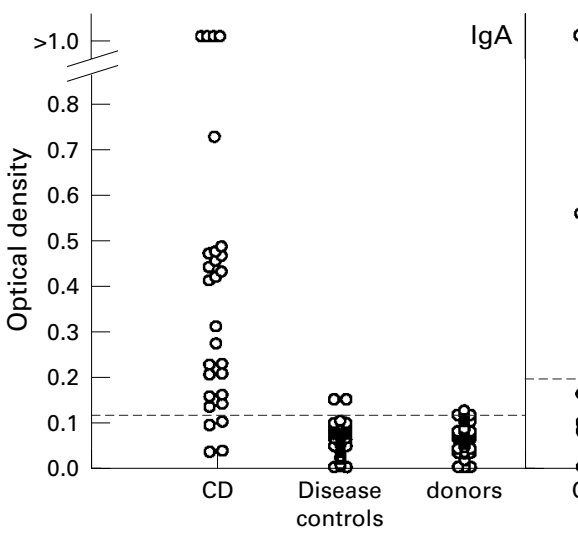

$\infty$ $\lg G$

Figure 1 A comparison of anti-tissue transglutaminase antibody values (IgA and IgG class) in patients with coeliac disease (CD), other gastrointestinal disorders (disease controls), and blood donors. The upper limit of normality is represented by the horizontal dotted line (optical density 0.12 for $\operatorname{Ig} A$ and 0.2 for $\operatorname{Ig} G$ ).
LABORATORY METHODS

Immunofluorescence methods and the antigliadin ELISA were performed as described before. ${ }^{1}$ Sera for immunofluorescence were screened at a $1 / 5$ dilution for IgA AEA (both monkey oesophagus and human umbilical cord as substrate) and 1/10 for IgA R1-ARA (rat liver, kidney, and stomach as substrate). Screening dilutions for anti-gliadin ELISA were $1 / 100$ for IgA antibody and $1 / 1000$ for IgG antibody.

The ELISA for tissue transglutaminase antibody (anti-TT) was based on, but different from, the method described by Dieterich et al. ${ }^{5}$ Following preliminary experiments using 1\% BSA, $1 \%$ human serum albumin (HSA), or no blocker, we elected not to use a block step, since BSA can represent a food antigen with the development of anti-BSA, and this could generate false positive results.

We used $100 \mu \mathrm{l}$ volumes of guinea pig TT (Sigma) at $10 \mu \mathrm{g} / \mathrm{ml}$ in phosphate buffered saline (PBS; $\mathrm{pH}$ 7.4) to coat the plates overnight at $4^{\circ} \mathrm{C}$. All further incubations used $100 \mu \mathrm{l}$ volumes, at room temperature, for one hour. All wash steps used $0.05 \%$ Tween/PBS repeated three times. After washing, patient's serum diluted to $1 / 100$ in $0.05 \%$ Tween/PBS was applied to duplicate wells. After a further wash, $100 \mu \mathrm{l}$ of either alkaline phosphatase conjugated goat anti-human IgA (Dako) diluted 1 in 300 or alkaline phosphatase conjugated goat anti-human IgG (Dako) diluted 1 in 1000 in $0.05 \%$ Tween/PBS was added to each well. Sigma 104 substrate was used according to the manufacturer's instructions, and the optical density (OD) read at 405 $n m$. Each patient's OD was corrected for reactivity of the patient's serum on test wells coated with PBS instead of TT solution. Appropriate positive and negative (no serum) controls were included.

We investigated the need to include calcium in the coating buffer. The above ELISAs were repeated using a range of concentrations up to $0.4 \mathrm{~g} / 1 \mathrm{CaCl}$ in TRIS saline as the coating buffer.

The upper limit of normal $(\mathrm{OD}=0.12$ for IgA, OD $=0.20$ for IgG) was established by determining the mean OD +2 SD for the 50 randomly selected healthy blood donor sera. IgA deficiency was excluded in the coeliac disease cases by nephelometry. Statistics quoted used the $\chi^{2}$ test with Yates correction.

\section{Results}

The percentage of coeliac disease patients and disease controls positive for each of the assays is shown in table 1, and the corresponding sensitivity/specificity data, calculated using coeliac disease versus disease control group, are shown in table 2. (The data from the blood donors were not used in calculating the data in table 2 as none of them had undergone small intestinal biopsy.) Sixteen of 23 IgA anti-TT positive coeliac disease sera were R1-ARA positive, but all were positive for IgA AEA (monkey oesophagus). One of the two anti-TT seropositive controls was a coeliac disease relative. The other was a case of unexplained anae- 
Table 3 Serological data on four patients with biopsy proved coeliac disease but negative for IgA anti-tissue transglutaminase (TT)

\begin{tabular}{|c|c|c|c|c|c|c|}
\hline \multirow[b]{2}{*}{ Patient } & \multicolumn{2}{|c|}{ Anti-gliadin antibodies } & \multirow{2}{*}{$\begin{array}{l}\text { Anti-endomysial } \\
\text { antibodies }{ }^{\star}\end{array}$} & \multirow[b]{2}{*}{ R1-anti reticulin } & \multicolumn{2}{|l|}{ Anti-TT } \\
\hline & $\operatorname{Ig} A$ & $\operatorname{Ig} G$ & & & $\operatorname{Ig} A$ & $\operatorname{Ig} G$ \\
\hline 1 & 21 & 48 & Negative & Negative & 0.094 & 0.228 \\
\hline 2 & 9 & 65 & $1 / 20$ & PP & 0.102 & 0.563 \\
\hline 3 & 35 & 21 & $1 / 40$ & Negative & 0.036 & 0.039 \\
\hline 4 & 41 & 21 & $1 / 10$ & Negative & 0.038 & 0.087 \\
\hline Normal range & $<15$ (units) & $<15$ (units) & Negative (titre) & Negative & $<0.120(\mathrm{OD})$ & $<0.200(\mathrm{OD})$ \\
\hline
\end{tabular}

PP, partial positive; *titres using human umbilical cord as substrate.

mia. Both had normal small bowel morphology, including normal intraepithelial lymphocyte count and normal villus to crypt ratio. High titre IgA anti-TT (OD > 0.2) was only seen in coeliac disease, but high titre IgG anti-TT (OD > 0.3) was found in all groups (fig 1). None of the four IgA anti-TT seronegative coeliac disease cases was IgA deficient. Quantitative serological data on these four sera are given in table 3 . The number of $\operatorname{IgA}$ anti-TT seropositive coeliac disease patients significantly exceeded the number of disease controls who were seropositive $(p<0.001)$.

We found that adding calcium to the coating buffer for the anti-TT ELISAs increased the signal, but also increased the background. The overall effect was that there was no improvement in the assay compared with the ELISA without calcium added. The between batch coefficient of variation was $7.4 \%$ for the IgA anti-TT ELISA (range $6.0 \%$ to $8.6 \%$ ) and $6.4 \%$ for the IgG anti-TT ELISA (range $4.3 \%$ to $8.3 \%$ ) (means of results from six samples repeated on 16 occasions).

Figure 2 shows IgA class antibody ELISA data from six sera using three different blockers-BSA, human serum albumin (HSA), or no blocker (PBS only). Sera from three coeliac disease patients (CD1, CD2, and

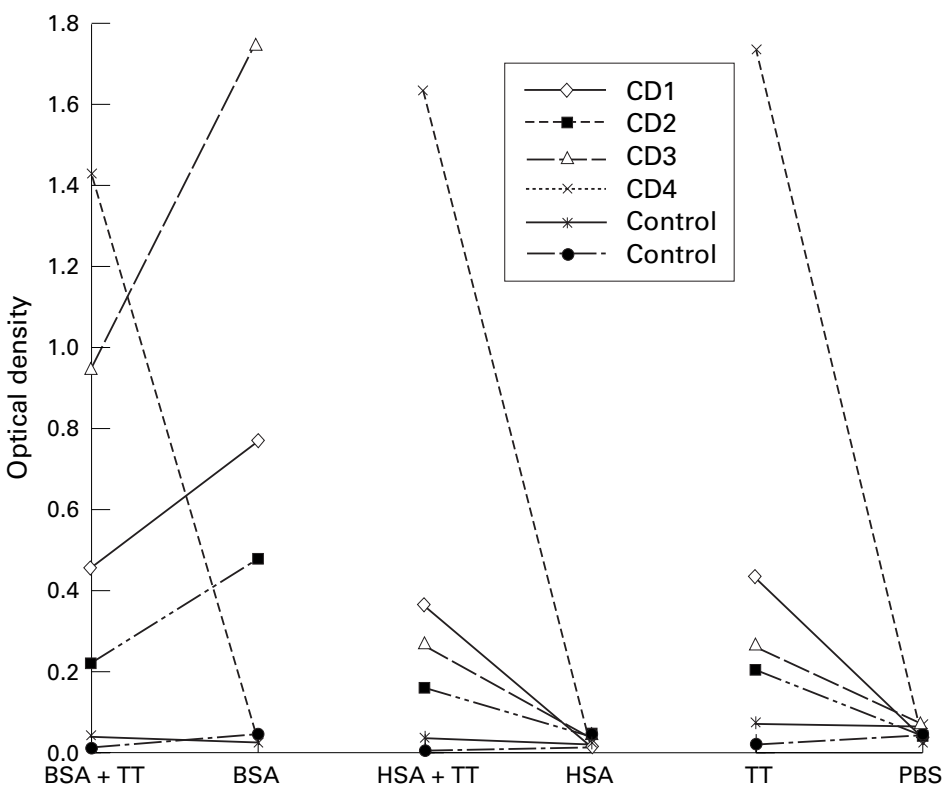

Figure 2 The effect of different blocking strategies on the ELISA for IgA anti-TT. BSA + TT: transglutaminase (TT) coated, bovine serum albumin (BSA) blocked; BSA: uncoated, BSA blocked; HSA + TT: TT coated, human serum albumin (HSA) blocked; HSA: uncoated, HSA blocked; TT: TT coated, unblocked (PBS only); PBS: uncoated, unblocked. Note that sera from coeliac disease patients CD1, CD2, and CD3 show strong reactivity with bovine serum albumin.
CD3) were found to have significant reactivity with BSA alone. CD4 illustrates the pattern seen in a sample with anti-TT activity without anti-BSA reactivity. Controls 1 and 2 are from disease control patients without either reactivity. Note typical background of approximately 0.05 OD units in uncoated wells.

\section{Discussion}

The sensitivity, specificity, and positive predictive values reported here for the established assays approximate closely to the experience reported by others as reviewed. ${ }^{1}$ Our $\operatorname{IgA}$ R1-ARA sensitivity is perhaps a little lower than the corresponding IgA AEA sensitivity because we used serum dilutions of $1 / 10$ and $1 / 5$, respectively. High titre anti-TT is clearly strongly associated with untreated coeliac disease. The exact relation between the antibodies (ARA, AEA, and anti-TT) detected in the various tests remains unclear. Dieterich et al absorbed anti-endomysial antibody with pure $\mathrm{TT}^{5}$ and one might predict that an ELISA method for detection of antibodies to TT would detect anti-endomysial antibody with an improved sensitivity. However, our results do not support this. "False" positives and "false" negatives were seen in our $\operatorname{IgA}$ anti-TT assay. One of the seropositive disease controls was a relative of a known coeliac, and may yet prove to have latent coeliac disease. However, given that our results point to superior sensitivity with the IgA AEA assay, concomitant AEA seropositivity might have been expected in a true case of latency, but this was not observed. None of the disease controls with abnormal small bowel mucosae was seropositive in any of the IgA based assays including IgA anti-TT. Hence, anti-TT generation as a consequence of non-specific mucosal damage or altered permeability seems unlikely. Dieterich et al reported false negatives with their IgG anti-TT assay, ${ }^{5}$ and we have confirmed this finding in our study. In addition we have detected false positive IgG anti-TT in both disease controls and normal donors.

We addressed two technical aspects of the original ELISA described by Dieterich et al. Our first concern was in the use of BSA as a blocking agent. We confirmed that sera from some coeliac disease patients do indeed have significant reactivity with BSA (fig 2) and therefore excluded this from all subsequent experiments. The second aspect was the question as to whether there is a need to use calcium to stabilise TT coating on the plates. TT is a calcium dependent enzyme and its conforma- 
tion may be altered in the presence of calcium. We found no improvement in disease sensitivity when calcium was incorporated (data not shown).

Several anti-TT seropositive samples were anti-R1-ARA negative, but this may reflect lower sensitivity of our R1-ARA test. Only 23/27 coeliac disease AEA positives and 23/26 umbilical cord AEA positives were also anti-TT positive. None was IgA deficient. The interim conclusion is that several distinct autoantibody specificities may exist. One of these, anti-TT, is clearly demonstrable in the majority of patients. The degree to which these specificities overlap requires further research.

As to the value of anti-TT tests in serological diagnosis, at least when using guinea pig antigen, the test is not better than the established IgA anti-endomysial antibody test, either in terms of sensitivity or specificity. Use of human TT or other technical modifications may yet improve the test reliability.

Funding for laboratory costs was kindly provided by Southmead Hospital "Showering Fund."

1 Unsworth DJ. Serological diagnosis of gluten-sensitive enteropathy. I Clin Pathol 1996;49:704-11.

2 Mäki M, Collin P. Coeliac disease (seminar). Lancet 1997;349:1755-9.

3 Unsworth DJ, Brown DL. Serological screening suggests that adult coeliac disease is underdiagnosed in the UK and increases the incidence by up to $12 \%$. Gut 1994;35:61-4.

4 Mäki M, Holm K, Lipsanen V, et al. Serological markers and HLA genes among healthy first-degree relatives of patients HLA genes among healthy first-degree relatives

5 Dieterich W, Ehnis T, Bauer M, et al. Identification of tissue transglutaminase as the autoantigen in celiac disease. Nature Med 1997;3:797-801.

6 Unsworth DJ, Manuel PD, Walker-Smith JA, et al. New Dis Child 1981;56:864-8. 